\title{
Correction to: A foliar Epichloë endophyte and soil moisture modified belowground arbuscular mycorrhizal fungal biodiversity associated with Achnatherum inebrians
}

\section{Rui Zhong • Chao Xia • Yawen Ju • Xingxu Zhang •}

Tingyu Duan • Zhibiao Nan • Chunjie Li

Published online: 4 May 2020

(C) Springer Nature Switzerland AG 2020

Correction to: Plant and Soil.

https://doi.org/10.1007/s11104-019-

04365-7.

The original version of this paper unfortunately contained errors, the spelling of one Latin name in some places was published incorrectly.

The "Epichloë gansusensis" should be modified to "Epichloë gansuensis". This has been corrected in this proof.

R. Zhong $\cdot$ C. Xia $\cdot$ Y. Ju $\cdot$ X. Zhang $(\bowtie) \cdot$ Z. Nan $(\bowtie) \cdot$

C. $\mathrm{Li}$

State key Laboratory of Grassland Agro-ecosystems, Key

Laboratory of Grassland Livestock Industry Innovation, Ministry

of Agriculture and Rural Affairs, Center for Grassland

Microbiome, College of Pastoral Agricultural Science and

Technology, Lanzhou University, Lanzhou 730020, People's

Republic of China

e-mail: xxzhang@lzu.edu.cn

e-mail: zhibiao@lzu.edu.cn 